\title{
BREMSSTRAHLUNG PAIR PRODUCTION IN RELATIVISTIC HEAVY ION COLLISION
}

\author{
Helmar Meier ${ }^{1}$, Kai Hencken ${ }^{1,2}$, Dirk Trautmann ${ }^{1}$, and Gerhard Baur ${ }^{3}$ \\ ${ }^{1}$ Institut für Physik der Universität Basel, 4056 Basel, Switzerland \\ ${ }^{2}$ National Institute of Nuclear Theory, University of Washington, Seattle, WA 98195, USA \\ ${ }^{3}$ Institut für Kernphysik (Theorie), Forschungszentrum Jülich, 52425 Jülich, Germany
}

(May 2, 2017)

\begin{abstract}
We calculate production of electron- and muon-pairs by the bremsstrahlung process in hadron collisions and compare it with the dominant two-photon process. Results for the total cross section are given for proton-proton and heavy-ion collisions at energies of the Relativistic Heavy Ion Collider (RHIC) and the Large Hadron Collider (LHC).
\end{abstract}

\section{INTRODUCTION}

Dileptons produced in central heavy ion collisions are investigated as one of the possible signal for the formation of the quark gluon plasma. The electromagnetic production of these leptons mainly in peripheral collisions is the dominant background process and therefore has to be studied in detail. Furthermore the production of muon pairs has been proposed as a possible way to measure the beam luminosity in the storage ring at the Large Hadron Collider (LHC) [1]. In order to be comparable with other methods the rate of muon pair production has to be known to better than one percent. A recent study shows [2], that inelastic photon emission processes, where the proton is excited to, e.g., a $\Delta$, already contribute at this level. In this article we want to study the importance of the bremsstrahlung process for the total rate.

Counting powers of $Z$ for different processes, one finds that the bremsstrahlung process is proportional to $Z^{6}$, that is, two powers of $Z$ enhanced compared to the dominant two-photon process $\left(\sim Z^{4}\right)$. Therefore one could expect this process to be at least of the same order of magnitude as the two-photon process for very heavy ions. But for high energies it was found to be much smaller. An estimate of the cross section was done in [3] and a more detailed calculation for $e^{-} e^{+}$collisions at LEPII energies can be found in 迎. The two processes lead to lepton pairs with different $C$-parity. Therefore the interference between the two mechanism vanishes for the total cross section. It was found that only for large scattering angles of the projectile the bremsstrahlung production is dominant (see [5] for a calculation of pair production from muon-nucleus collisions). Large scattering angles do not contribute much to the total cross sections and for nuclei they are further reduced due to the finite size. Collisions with large momentum transfer are very likely to breakup the nucleus; the elastic form factor falls off quickly for large $q^{2}$.

\section{LEPTON PAIR PRODUCTION}

The cross section for the two-photon pair production has been calculated in the semi-classical approximation in [6], where the fields of the colliding ions have been treated as external fields using also the straight line approximation. Small impact parameters, smaller than twice the nuclear radius, where the ions interact strongly, have been cut off. Whereas the analysis of [6] was mainly on studying the production of multiple (electron-positron) pairs, here we are considering only single-pair creation.

The cross section for the bremsstrahlung pair production has been calculated in lowest order perturbation theory [7, 8. One of the corresponding Feynman diagrams is shown in Fig. 1.

FIG. 1. A Feynman diagram of bremsstrahlung pair production.

Using 'Heaviside-convention', that is,

$$
\alpha=\frac{e^{2}}{4 \pi \hbar c}
$$

the S-matrix element is given by 


$$
\begin{aligned}
S_{f i}= & i \frac{Z_{A}^{2} Z_{B} e^{4}}{V^{3}} \sqrt{\frac{M_{A}^{2} M_{B}^{2} m^{2}}{E_{f}^{A} E_{i}^{A} E_{f}^{B} E_{i}^{B} E^{e^{-}} E^{e^{+}}}} \\
& \times(2 \pi)^{4} \delta^{4}\left(p_{f}^{A}+p_{f}^{B}+p^{e^{-}}+p^{e^{+}}-p_{i}^{A}-p_{i}^{B}\right) \\
& \times\left\{\frac{1}{\left(p^{e^{-}}+p^{e^{+}}\right)^{2}+i \varepsilon} \frac{1}{\left(p_{i}^{B}-p_{f}^{B}\right)^{2}+i \varepsilon}\right. \\
& \times\left[\bar{u}\left(p_{f}^{A}, s_{f}^{A}\right) \gamma_{\mu} \frac{1}{\not p_{f}^{A}+\not p^{-}+\not e^{+}-M_{A}+i \varepsilon} \gamma_{\nu} u\left(p_{i}^{A}, s_{i}^{A}\right)\right. \\
& \times \bar{u}\left(p^{e^{-}}, s^{e^{-}}\right) \gamma^{\mu} v\left(p^{e^{+}}, s^{e^{+}}\right) \times \bar{u}\left(p_{f}^{B}, s_{f}^{B}\right) \gamma^{\nu} u\left(p_{i}^{B}, s_{i}^{B}\right) \\
& +\bar{u}\left(p_{f}^{A}, s_{f}^{A}\right) \gamma_{\mu} \frac{1}{\not p_{f}^{A}+\not p_{f}^{B}-\not p_{i}^{B}-M_{A}+i \varepsilon} \gamma_{\nu} u\left(p_{i}^{A}, s_{i}^{A}\right) \\
& \left.\left.\times \bar{u}\left(p_{f}^{B}, s_{f}^{B}\right) \gamma^{\mu} u\left(p_{i}^{B}, s_{i}^{B}\right) \times \bar{u}\left(p^{e^{-}}, s^{e^{-}}\right) \gamma^{\nu} v\left(p^{e^{+}}, s^{e^{+}}\right)\right]\right\},
\end{aligned}
$$

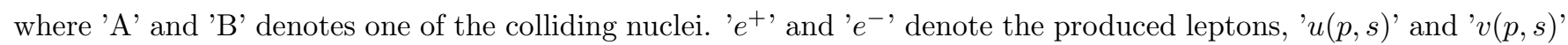
are the usual Dirac spinors for the plane wave solution. Describing the heavy ions as spin- $1 / 2$ particles corresponds to giving them a magnetic moment corresponding to $g=2$, much larger than their experimental value. But due to their large mass, spin dependent terms, which are of order $1 / M$, are effectively suppressed. Therefore our calculation is really independent of the real spin of the nuclei and is valid also for spin-0 nuclei. We are taking the finite size of the two nuclei into account by using elastic form factors (eqs. (6) and (耳)). The cross section is given by

$$
\begin{aligned}
\sigma= & \int \frac{M_{A} M_{B}}{\sqrt{\left(p_{i}^{A} p_{i}^{B}\right)^{2}-M_{A}^{2} M_{B}^{2}}}\left|\bar{M}_{f i}\right|^{2} \\
& \times(2 \pi)^{4} \delta^{4}\left(p_{f}^{A}+p_{f}^{B}+p^{e^{-}}+p^{e^{+}}-p_{i}^{A}-p_{i}^{B}\right) \\
& \times \frac{M_{A} d^{3} p_{f}^{A}}{(2 \pi)^{3} E_{f}^{A}} \frac{M_{B} d^{3} p_{f}^{B}}{(2 \pi)^{3} E_{f}^{B}} \frac{m d^{3} p^{e^{-}}}{(2 \pi)^{3} E^{e^{-}}} \frac{m d^{3} p^{e^{+}}}{(2 \pi)^{3} E^{e^{+}}}
\end{aligned}
$$

with the matrix element squared

$$
\begin{aligned}
& \left|\bar{M}_{f i}\right|^{2}=\frac{1}{4} Z_{A}^{4} Z_{B}^{2} e^{8} \frac{1}{\left(p^{e^{-}}+p^{e^{+}}\right)^{4}} \frac{1}{\left(p_{i}^{B}-p_{f}^{B}\right)^{4}} \\
& \times \operatorname{Tr} \frac{\not p^{e^{-}}+m}{2 m} \gamma_{\mu} \frac{\not p^{+}+m}{2 m} \gamma_{\sigma} \\
& \times \operatorname{Tr} \frac{\not p_{f}^{B}+M_{B}}{2 M_{f}^{B}} \gamma_{\nu} \frac{\not p_{i}^{B}+M_{B}}{2 M_{B}} \gamma_{\lambda} \\
& \times\left(\operatorname{Tr} \frac{\not p_{f}^{A}+M_{A}}{2 M_{A}} \gamma^{\mu} \frac{\not p_{f}^{A}+\not p^{e^{-}}+\not p^{e^{+}}+M_{A}}{\left(p_{f}^{A}+p^{e^{-}}+p^{e^{+}}\right)^{2}-M_{A}^{2}} \gamma^{\nu} \frac{\not p_{i}^{A}+M_{A}}{2 M_{A}} \gamma^{\lambda} \frac{\not p_{f}^{A}+\not p^{e^{-}}+\not p^{e^{+}}+M_{A}}{\left(p_{f}^{A}+p^{e^{-}}+p^{e^{+}}\right)^{2}-M_{A}^{2}} \gamma^{\sigma}\right. \\
& +\operatorname{Tr} \frac{\not p_{f}^{A}+M_{A}}{2 M_{A}} \gamma^{\mu} \frac{\not \not_{f}^{A}+\not p^{e^{-}}+\not p^{e^{+}}+M_{A}}{\left(p_{f}^{A}+p^{e^{-}}+p^{e^{+}}\right)^{2}-M_{A}^{2}} \gamma^{\nu} \frac{\not p_{i}^{A}-M_{A}^{2}}{2 M_{A}} \gamma^{\sigma} \frac{\not p_{f}^{A}+\not p_{f}^{B}-\not p_{i}^{B}+M_{A}}{\left(p_{f}^{A}+p_{f}^{B}-p_{i}^{B}\right)^{2}-M_{A}^{2}} \gamma^{\lambda} \\
& +\operatorname{Tr} \frac{\not p_{f}^{A}+M_{A}}{2 M_{A}} \gamma^{\nu} \frac{\not p_{f}^{A}+\not p_{f}^{B}-\not p_{i}^{B}+M_{A}}{\left(p_{f}^{A}+p_{f}^{B}-p_{i}^{B}\right)^{2}-M_{A}^{2}} \gamma^{\mu} \frac{\not p_{i}^{A}+M_{A}}{2 M_{A}} \gamma^{\lambda} \frac{\not p_{f}^{A}+\not p^{e^{-}}+\not p^{e^{+}}+M_{A}}{\left(p_{f}^{A}+p^{e^{-}}+p^{e^{+}}\right)^{2}-M_{A}^{2}} \gamma^{\sigma} \\
& \left.+\operatorname{Tr} \frac{\not p_{f}^{A}+M_{A}}{2 M_{A}} \gamma^{\nu} \frac{\not p_{f}^{A}+\not p_{f}^{B}-\not \not_{i}^{B}+M_{A}}{\left(p_{f}^{A}+p_{f}^{B}-p_{i}^{B}\right)^{2}-M_{A}^{2}} \gamma^{\mu} \frac{\not p_{i}^{A}+M_{A}}{2 M_{A}} \gamma^{\sigma} \frac{\not p_{f}^{A}+\not p_{f}^{B}-\not \not_{i}^{B}+M_{A}}{\left(p_{f}^{A}+p_{f}^{B}-p_{i}^{B}\right)^{2}-M_{A}^{2}} \gamma^{\lambda}\right) .
\end{aligned}
$$

In eq. (2) and (14) we only consider the case, where ion 'A' emits the virtual (space-like) photon, that creates the pair. To this we have to add the diagrams, where the virtual photon is emitted by ion 'B'. Furthermore as the ions are identical, we have to add the contribution from the exchange diagrams. At high energies the interference terms between these different processes are completely negligible. In order to be significant they must have a sufficient overlap in the final phase space. We can estimate their importance by comparing the product of the absolute value of the matrix elements with the cross section of each of them. This estimation shows that we can neglect all interference effects. We get the total cross section by multiplying eq. (3) by a factor of two, which accounts for the photon emission from ion 'B'. We calculate the trace in eq. (4) with the help of the algebraical calculation program FORM [9]. 


\section{THE FORM FACTORS}

As mentioned in the previous section, we have to take into account the finite size of the nuclei. We introduce a form factor at each vertex in the Feynman diagram. This changes the equation of the absolute value squared of the matrix element in in eq. (4) to

$$
\left|\bar{M}_{f i}\right|^{2} \rightarrow\left|\bar{M}_{f i}\right|^{2}\left|F_{A}\left(q_{1}^{2}\right)\right|^{2}\left|F_{B}\left(q_{1}^{2}\right)\right|^{2}\left|F_{T}\left(q_{2}^{2}\right)\right|^{2} .
$$

We need both space-like and time-like form factors for the nuclei. ' $q_{1}$ ' is the momentum transferred between nuclei 'A' and 'B' and is therefor space-like $\left(q_{1}^{2}<0\right)$. ' $q_{2}$ ' is the momentum of the virtual photon creating the pair; as $q_{2}^{2}$ is identical to the invariant mass of the lepton pair, it has to be at least $4 \mathrm{~m}_{\text {lepton }}^{2}$. Therefore it is time-like $\left(q_{2}^{2}>0\right)$.

In the space-like region we use the usual dipole form factor for proton-proton collisions

$$
F_{p}\left(q_{1}^{2}\right)=\left(\frac{1}{1+\frac{\left|q_{1}^{2}\right|}{\Lambda^{2}}}\right)^{2} \quad ; \Lambda^{2}=0.71 \mathrm{GeV}^{2}
$$

and for heavy ion collisions a Gaussian form factor

$$
F_{h . i .}\left(q_{1}^{2}\right)=\exp \left(-\frac{\left|q_{1}^{2}\right|}{\lambda^{2}}\right)
$$

with $\lambda^{2}=\frac{6}{\left\langle r^{2}\right\rangle}$ and $\sqrt{\left\langle r^{2}\right\rangle}=1.0 A^{\frac{1}{3}} \mathrm{fm}$, where $A$ is the mass number of the nucleus. The additional effect of the elastic form factor is that they suppress collisions with a large $q$-value, corresponding to small impact parameter $b$. Therefore we do not correct for impact parameter smaller than $2 R$, where both nuclei interact strongly. This effect could be included in principle within a Glauber model approach but is technically rather involved. In addition our calculation (neglecting this effect), gives an upper limit of the true cross section.

In the time-like region we make use of vector meson dominance (VMD), that is, we assume that the photon couple to the hadrons through vector mesons only. We use a form factor $F_{V M D}$ as discussed in 10]. As done there, we neglect the dependence due to the fact that the intermediate nucleus is off the mass-shell. $\overrightarrow{W e}$ do not sum over all intermediate states, taking only the ground state as intermediate state into account. In addition we don't take the absorption of the meson inside the nucleus into account. Both effects reduce the total cross section [11], therefore our calculation is again an upper limit.

$$
F_{T}\left(q_{2}^{2}\right) \approx F_{V M D}\left(q_{2}^{2}\right)=\frac{m_{V}^{2}}{m_{V}^{2}-q_{2}^{2}}
$$

Here $V$ is one the different light vector mesons ( $\rho$-, $\omega$ - or $\phi$-meson) through which the photon couples to the nucleus. In our calculations we only include the VMD form factor for the $\omega$-meson. The complex mass $m_{V}$ is then given by

$$
m_{V}=m_{\omega}-\frac{i \Gamma_{\omega}}{2}
$$

with $m_{\omega}=782 \mathrm{MeV}$ is the $\omega$-meson and $\Gamma_{\omega}=8.4 \mathrm{MeV}$ is its full decay width 12.

Due to this form of the form factor the differential cross section of the bremsstrahlung process has a very narrow resonance-like peak for invariant masses near the $\omega$ mass. As the form factor assume that the vector mesons couples coherently with the whole nucleus, this form factor is an upper limit for the VMD contribution. For heavy ions it is very unlikely that the vector meson couples coherently to all the nucleons due to their large size. Therefore we make use of the VMD only for proton-proton collisions, neglecting it completely for the heavy ion case. Using on the other hand the cross section as a function of the invariant mass (see Figs. 3 and 7 ) it is easy to calculate the total cross section for any realistic form factor.

We treat the two cases of electron-positron and muon-antimuon pair production separately. For electron-positron pair production the differential cross section $\left(=\frac{d \sigma}{d M_{\gamma}}\right)$ for invariant masses equal to vector meson masses is too small to contribute significantly to the total cross section (see Fig. 2 and Fig. 31). In contrast to this they are important for the muon-antimuon pair production as their mass is comparable to them. 


\section{RESULTS AND CONCLUSIONS}

The total cross sections are obtained from eq. (3) by a Monte Carlo integration over all possible final states (VEGAS [13]). As the phase-space for this four particle final state is rather restricted, we have chosen integration variable so that we integrate only over the allowed (physical) phase-space using the variables given in [14]. For this we split the four-particle phase-space into a chain of successive two particle decays integrating over the invariant mass of the intermediate states and the angles in the center of mass of the decaying particle. Momenta therefore have to be boosted from one center of mass system to the next one. Using these adequate phase space variables, the Monte Carlo integration can be done effectively.

\section{A. Total cross sections}

We give the total cross sections for the bremsstrahlung production and the corresponding two-photon process for electron-positron $\left(\sigma_{e^{-} e^{+}}\right)$and muon-pairs $\left(\sigma_{\mu^{-} \mu^{+}}\right)$for RHIC and LHC energies in tables II and II. The beam energy is giving in terms of the Lorentz gamma factor $E=\gamma M$ in the collider reference frame (center of mass of the two ions). We have calculated results for different ion species: ${ }_{20}^{40} \mathrm{Ca},{ }_{82}^{208} \mathrm{~Pb}$ and ${ }_{79}^{197} \mathrm{Au}$. As described above the form factor $\left(F_{V M D}\right)$ is used in the calculation for the results marked as 'Proton (VMD)'.

The total cross sections of bremsstrahlung production are compared with the results for two-photon production, also shown in tables II and II.

\section{B. Differential cross sections of bremsstrahlung pair production}

Figures 2 to 9 show the results for the differential cross sections as a function of the invariant mass of the produced pair $\left(\frac{d \sigma}{d M_{\gamma}}\right)$ and also as a function of the angle with respect to the beam $\left(\frac{d \sigma}{d \theta}\right)$. We do not show results using the VMD form factor. The effect of this form factor can be easily found by multiplying the cross section with the absolute value square of it (see Eqs. (5) and (8)). It results in a peaks around $q_{2}^{2} \approx m_{\omega}^{2}$ in the differential cross section $\frac{d \sigma}{d M_{\gamma}}$.

FIG. 2. The cross section $\sigma$ as a function of the mass of the virtual photon $M_{\gamma}\left(=\frac{d \sigma}{d M_{\gamma}}\right)$ which creates the electron-positron pair. The solid line are the results for a lead beam, the dot-dashed line for a calcium beam and the dashed line for a proton beam at LHC energies.

FIG. 3. The cross section $\sigma$ as a function of the mass of the virtual photon $M_{\gamma}$ which creates the electron-positron pair $\left(=\frac{d \sigma}{d M_{\gamma}}\right)$. The solid line are the results for a gold beam and the dashed line for a proton beam at RHIC energies.

FIG. 4. The cross section $\sigma$ as a function of the scattering angle $\theta$ of the created electron (or positron) with the beam axis $\left(=\frac{d \sigma}{d \theta}\right)$. The solid line are the results for a lead beam, the dot-dashed line for a calcium beam and the dashed line for a proton beam at LHC energies.

FIG. 5. The cross section $\sigma$ as a function of the scattering angle $\theta$ of the created electron (or positron) with the beam axis $\left(=\frac{d \sigma}{d \theta}\right)$. The solid line are the results for a gold beam and the dashed line for a proton beam at RHIC energies.

FIG. 6. same as Fig. 2 but for muon-antimuon pair production

FIG. 7. same as Fig. 3 but for muon-antimuon pair production

FIG. 8. same as Fig. 4 but for muon-antimuon pair production 
FIG. 9. same as Fig. 5 but for muon-antimuon pair production

\section{Conclusions}

Comparing the results for bremsstrahlung and two-photon production in tables If and II we see that the bremsstrahlung production contributes only by $<3 \times 10^{-3}$ to the rate of the created pairs by electromagnetic processes in heavy ion collision. Please have also in mind that our calculation is an upper limit of this cross section, therefore the real cross section is expected to be even smaller. So the additional factor $Z^{2}$ in the cross section cannot make up for the almost divergent photon propagators in two-photon case. The matrix elements of the two-photon process contain denominators which are very close to zero. This corresponds to the exchange of quasi real photons and does not occur for the bremsstrahlung case. The differential cross section for bremsstrahlung production without using the VMD form factor is also small compared to the dominant two-photon case. If one includes the VMD form factor, one get a peak in $\frac{d \sigma}{d M_{\gamma}}$ around the $\omega$-meson mass. The peaks are absent in the two-photon case. This could be used as a way to get more insight into the VMD contribution to this process and especially the time-like form factor of the proton and of nuclei. With respect to the use of muon pairs as a luminosity monitor for the LHC, we see that the bremsstrahlung production seems to be too small to be of importance at the $1 \%$ level. The largest uncertainty in our calculations is the almost unknown time-like form factor of proton and nuclei. Another source of uncertainty is the contribution coming from intermediate states different then the proton or the nucleus in its ground state. I has been proposed recently 15 to study the interference of virtual Compton scattering and the Bethe-Heitler process as a way to extract nucleon properties, that is, the form factor for the lepton pair production in virtual Compton scattering. The result of such a measurement can be easily used to improve our calculations.

\section{ACKNOWLEDGMENTS}

This work was supported in part by the Swiss National Science Foundation (SNF), the "Freiwillige Akademische Gesellschaft" (FAG) of the University of Basel, and the "Deutsche Forschungsgemeinschaft" (DFG). One of us (K.H.) would like to thank them for their financial support.

[1] K.Eggert, A.Morsch: LHC Note 264 CERN AT/94-05 (unpublished)

[2] O.Conradt: Äquivalente Photonenspektren und ihre Anwendung in der der elektromagnetischen Müonenpaarproduktion in peripheren pp-Kollision: Universität Basel, Diplomarbeit 1996 (unpublished)

[3] V.M.Budnev, I.F.Ginzburg, G.V.Meledin, V.R.Serbo: Nuclear Physics B 63 (1973) 519

[4] O.Panella, J.Parisi, P.Kessler: Photon 95: Wold Scientific (1995) 287

[5] S.J.Brodsky and S.C.C.Ting: Physical Review 145 (1966) 1018

[6] A.Alscher, K.Hencken, D.Trautmann, G.Baur: Physical Review A 55(1) (1997) 396 and references there

[7] J.D.Bjorken, S.D.Drell: Relativistische Quantenmechanik: B.I.-Hochschultaschenbuch 1966

[8] V.B.Berestetskii, E.M.Lifschitz, L.P.Pitaevskii: Quantum Elektrodynamics. Course of Theoretical Physics Vol.4: Butterworth-Heinemann 1996

[9] J.A.M.Vermaseren: 'Form' is an algebraical calculation program, the free version 1.0 can be found, e.g., at FTP.NIKHEF.NL.

[10] M.Schäfer, H.C.Dönges, U.Mosel: Physics Letters B 342 (1995) 13

[11] E.L.Bratovskaya, A.I.Titov, B.L.Renznik: Soviet Journal of Nuclear Physics 55(11) (1992) 1709

[12] R.M.Barnett et al: Rewiew of Particle Physics: Physical Rewiew D54 (1996) 1

[13] G.P.Lepage: 'Vegas': An algorithm for adaptive multidimensional integration: J.Comp.Phys. 27 (1978) 192 and Cornell laboratory for nuclear sciences report No. CLNS-80/ 477, 1980 (unpublished)

[14] E.Byckling,K.Kajantie: Particle Kinematics: John Wiley \& Sons 1973

[15] A.Yu.Korchin, O.Scholten, F. de Jong: Physics Letters B 402 (1997) 1

TABLE I. The total cross section of electron-positron pair production by bremsstrahlung (brems. p.p) and in two-photon physics (two phot.phy. p.p.) at RHIC and LHC energies for colliding protons, calcium, lead or gold nuclei. 


\begin{tabular}{lcccc} 
& $\gamma_{\text {collider }}$ & $\sigma_{e^{-} e^{+}}^{\text {brems. p.p. }}($ barn $)$ & $\sigma_{e^{-} e^{+}}^{\text {two phot. phy. p.p. }}($ barn $)$ & $\frac{\sigma_{e^{-}+}(\text {brems. p.p. })}{\sigma_{e^{-} e^{+}} \text {(two phot. phy.p.p. }}$ \\
\hline gold & 100 & $3.08 * 10^{-4}$ & $2.74 * 10^{4}$ & $1.1 * 10^{-8}$ \\
proton (w/o VMD) & 200 & $3.52 * 10^{-11}$ & $1.33 * 10^{-3}$ & $2.7 * 10^{-8}$ \\
lead & 2900 & $8.73 * 10^{-4}$ & $2.05 * 10^{5}$ & $4.3 * 10^{-9}$ \\
calcium & 3700 & $4.33 * 10^{-6}$ & $8.37 * 10^{2}$ & $5.2 * 10^{-9}$ \\
proton & 7500 & $5.88 * 10^{-11}$ & $6.69 * 10^{-3}$ & $8.8 * 10^{-9}$ \\
proton (VMD) & 7500 & $5.88 * 10^{-11}$ & $6.69 * 10^{-3}$ & $8.8 * 10^{-9}$ \\
\hline \hline
\end{tabular}

TABLE II. (as TABLE I, but for muon-antimuon pair production)

\begin{tabular}{lcccc}
\hline \hline & $\gamma_{\text {collider }}$ & $\sigma_{\mu_{-} \mu_{+}}^{\text {brems.p.p. }}($ barn $)$ & $\sigma_{\mu^{-} \mu^{+}}^{\text {two phot. phy. p.p. }(\text { barn })}$ & $\frac{\sigma_{\mu-\mu}(\text { brems. p.p. })}{\sigma_{\mu}-\mu^{+}(\text {two phot. phy. p.p. })}$ \\
\hline gold & 100 & $4.77 * 10^{-5}$ & $4.66 * 10^{-1}$ & $1.0 * 10^{-4}$ \\
proton & 200 & $1.42 * 10^{-12}$ & $3.14 * 10^{-8}$ & $4.5 * 10^{-5}$ \\
proton (VMD) & 200 & $2.8 * 10^{-10}$ & $3.14 * 10^{-8}$ & $2.7 * 10^{-3}$ \\
lead & 2900 & $2.02 * 10^{-4}$ & $4.60 * 10^{0}$ & $4.4 * 10^{-5}$ \\
calcium & 3700 & $7.76 * 10^{-7}$ & $1.78 * 10^{-2}$ & $4.4 * 10^{-5}$ \\
proton & 7500 & $2.80 * 10^{-12}$ & $1.36 * 10^{-7}$ & $2.0 * 10^{-5}$ \\
proton (VMD) & 7500 & $5.7 * 10^{-10}$ & $1.36 * 10^{-7}$ & $1.2 * 10^{-3}$ \\
\hline \hline
\end{tabular}




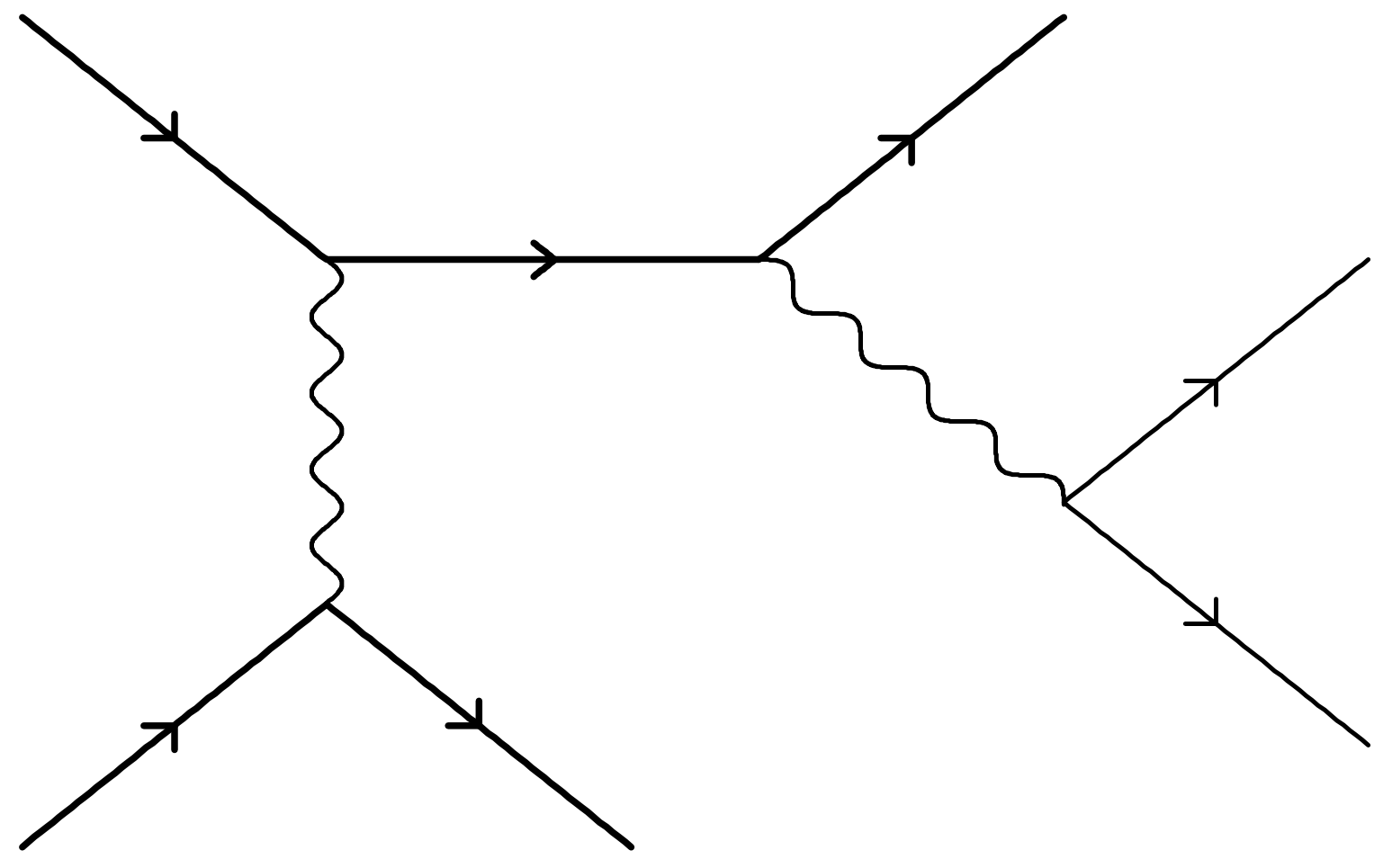




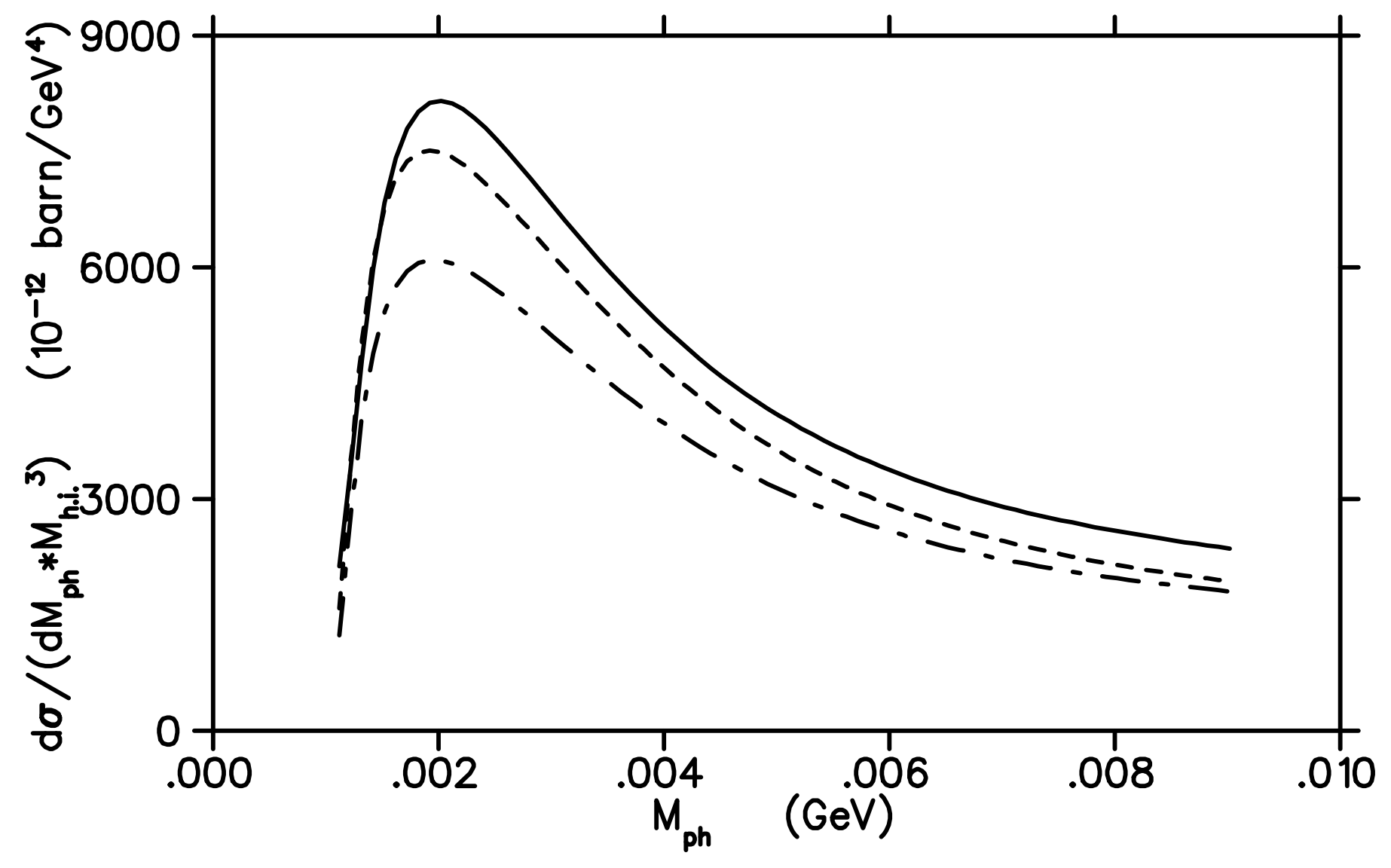




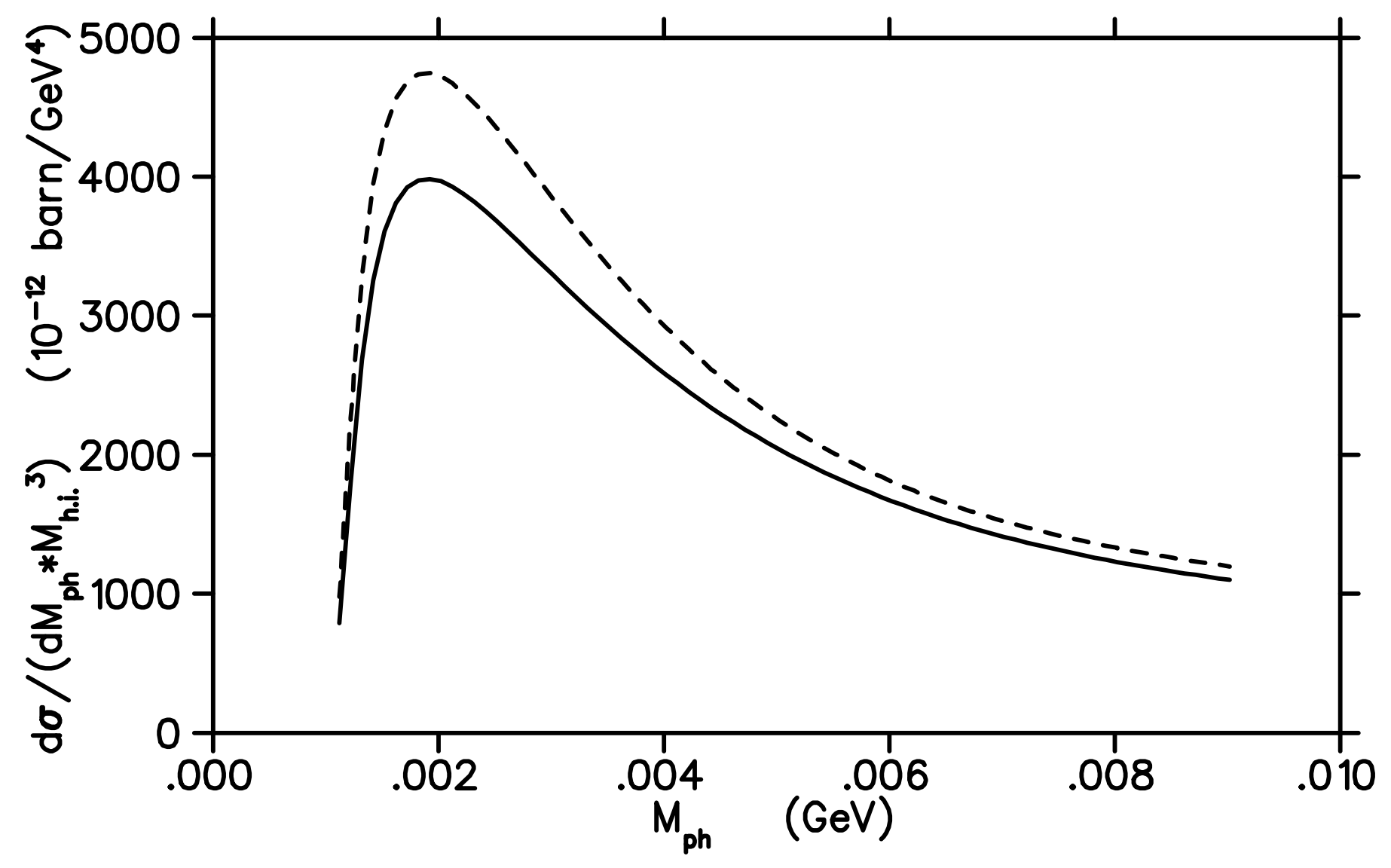




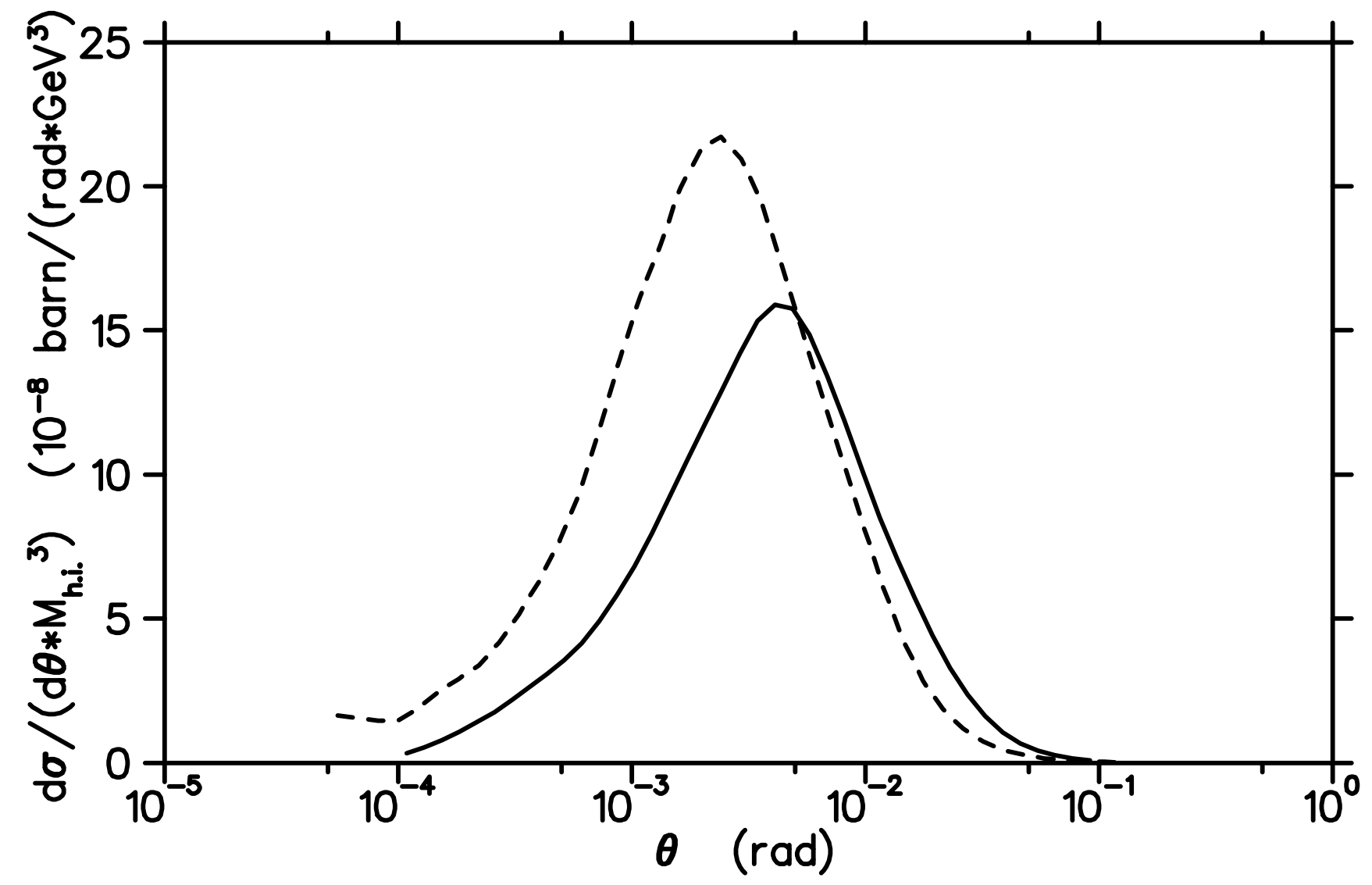




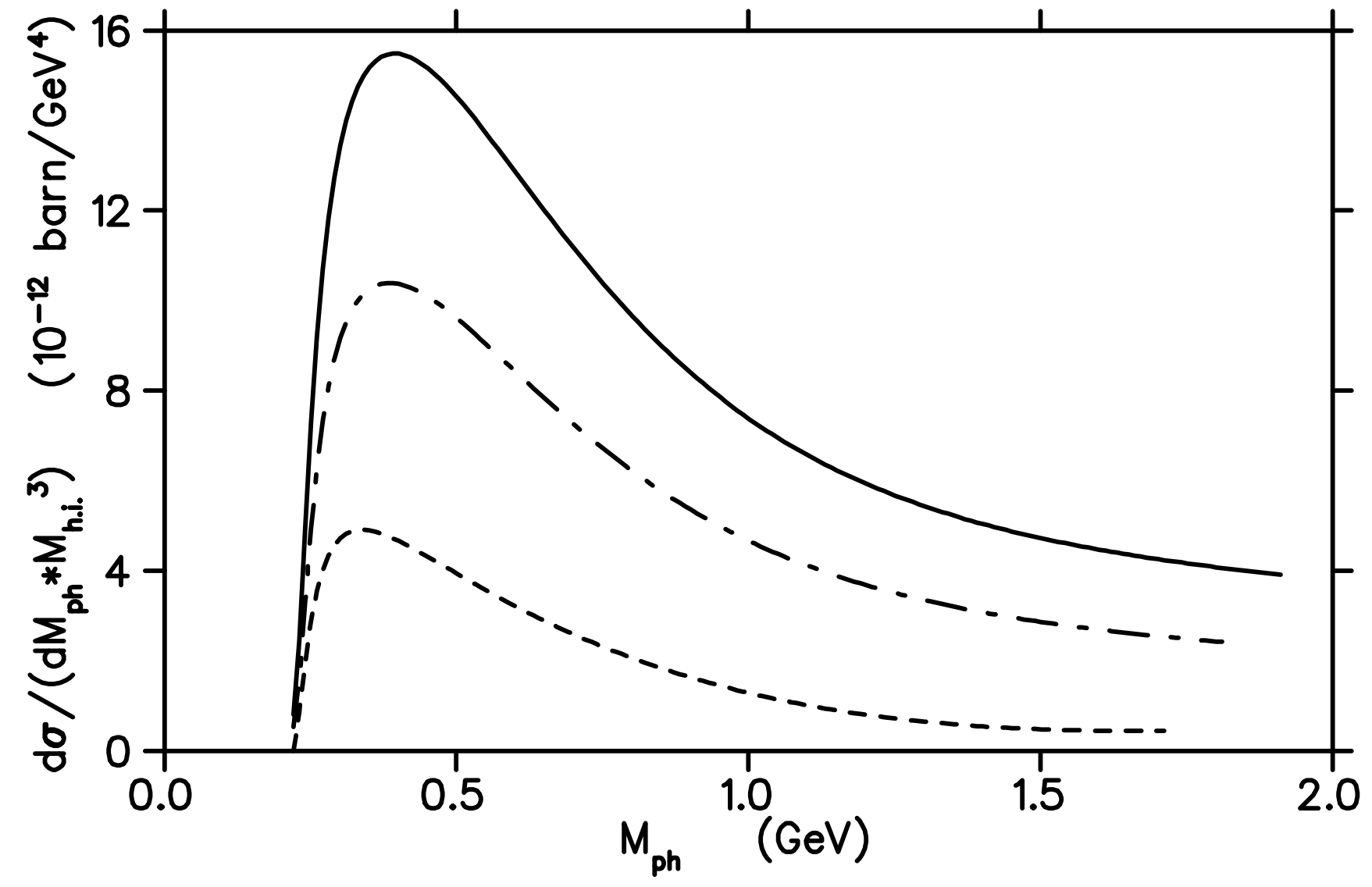




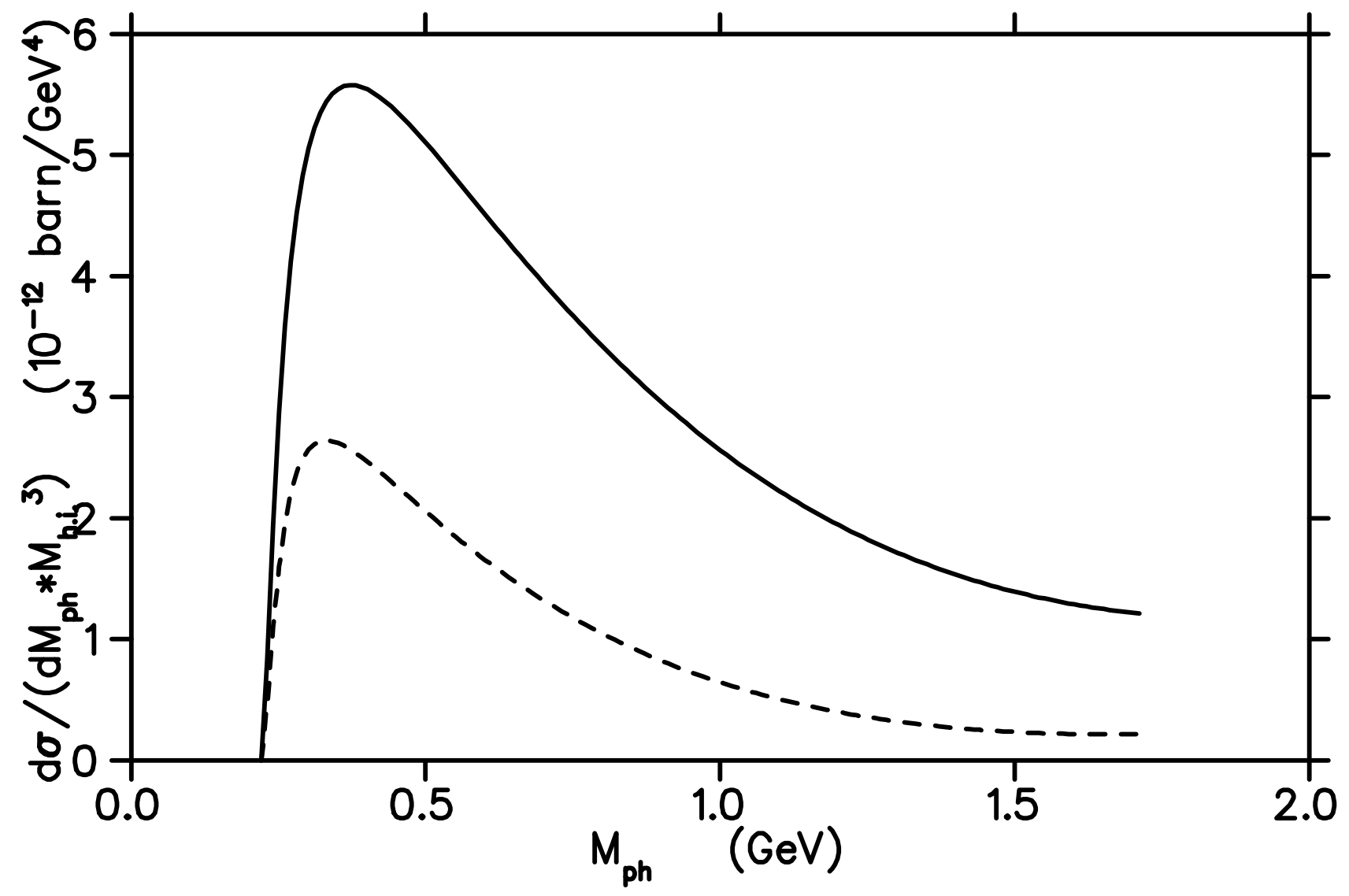




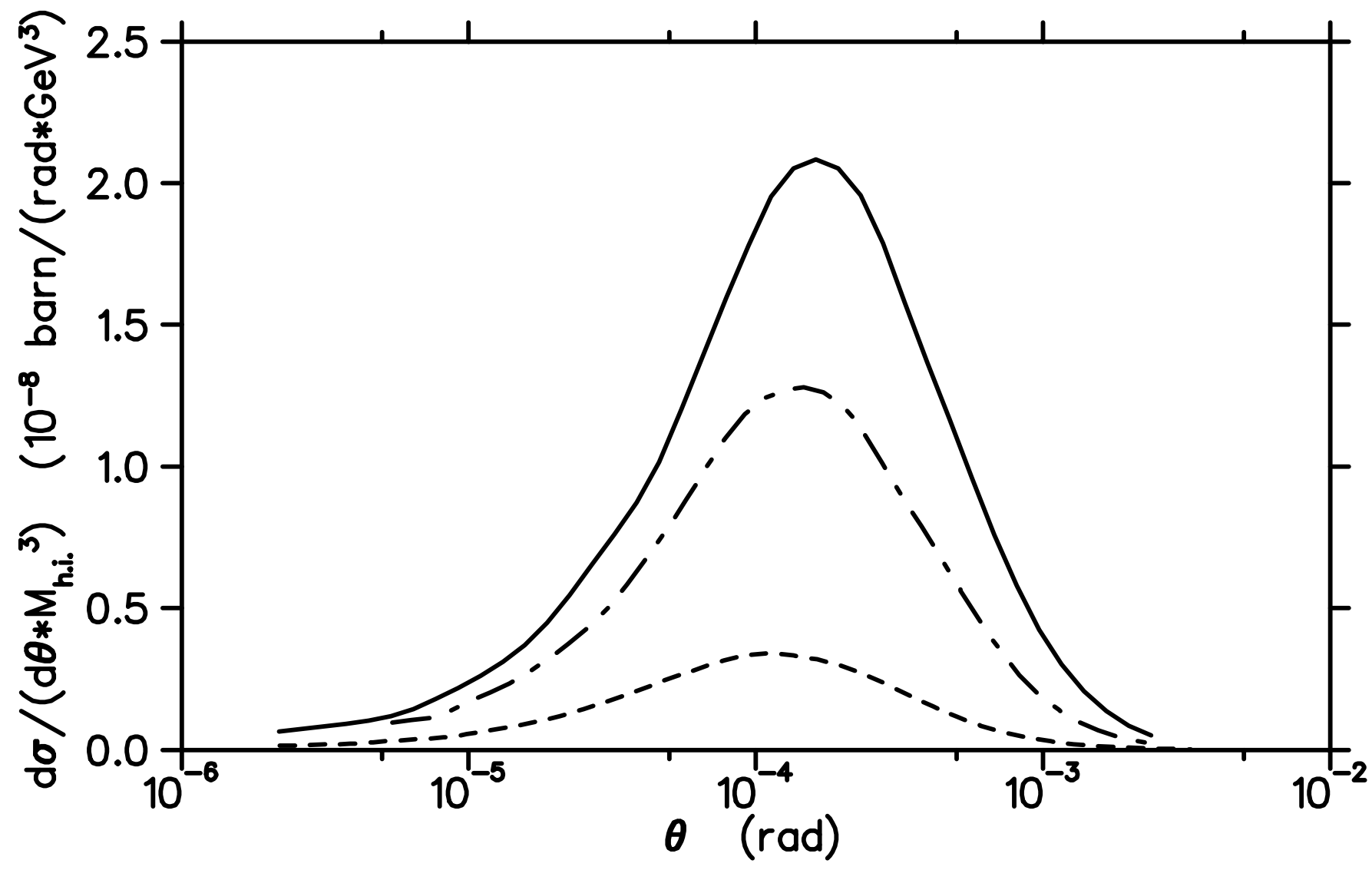




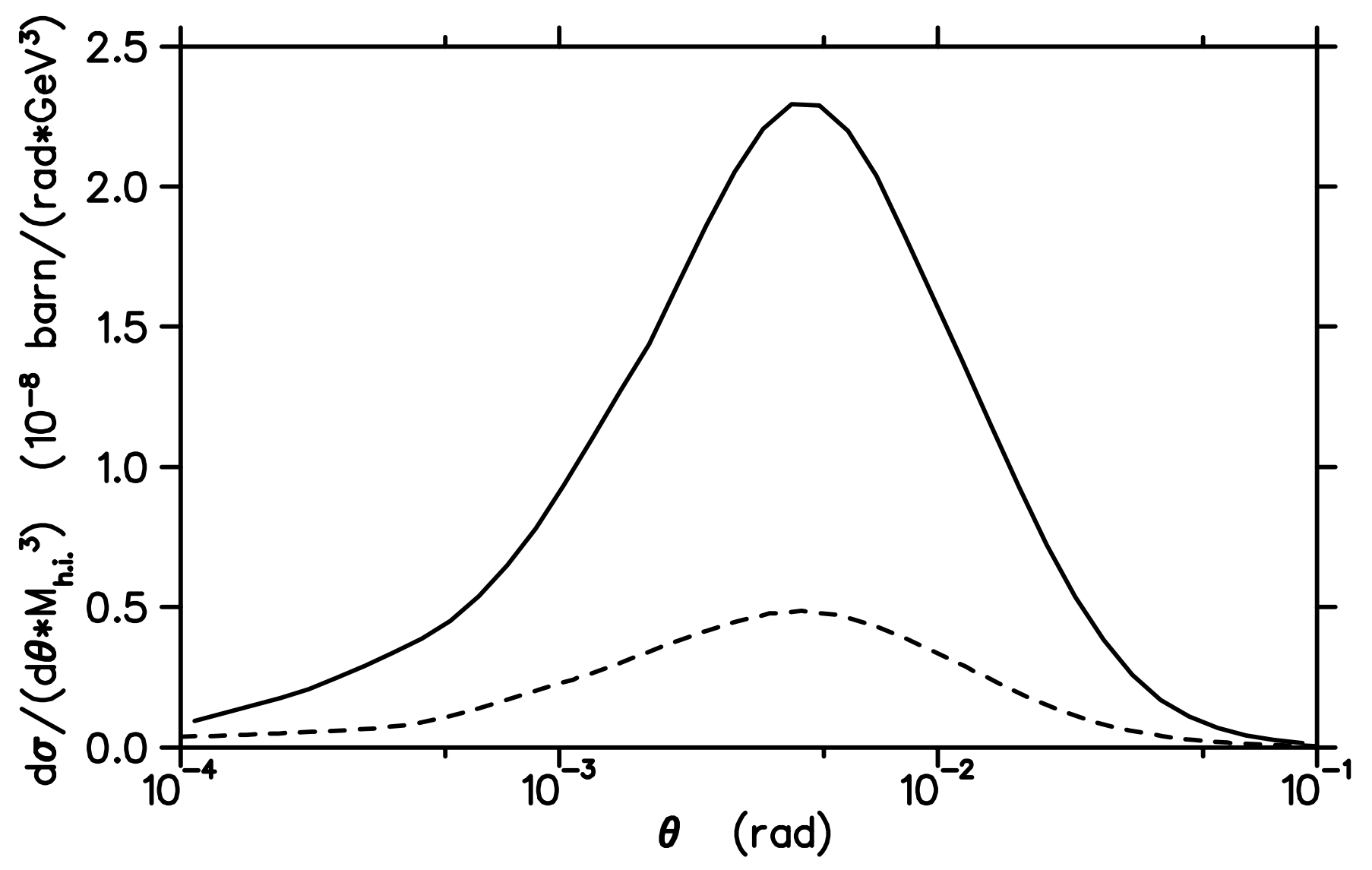

